\title{
The Improvement of V-Shaped Transfer Function of Binary Particle Swarm Optimization
}

\author{
Dong-Yang Zhang ${ }^{1,2}$, Jian-Hua Liu ${ }^{1,2(凶)}$, Lei Jiang ${ }^{1,2}$, Guan-Nan Bu ${ }^{1,2}$, \\ Ren-Yuan $\mathrm{Hu}^{1,2}$, and Yi-Xuan $\mathrm{Luo}^{1,2}$ \\ 1 School of Information Science and Engineering, Fujian University of Technology, \\ Fuzhou, China \\ jhliu@fjnu.edu.cn \\ ${ }^{2}$ Fujian Provicial Key Laboraty of BigData Mining and Application, Fuzhou, China
}

\begin{abstract}
Binary Particle Swarm Optimization (BPSO) is a swarm intelligence to optimize discrete space problems by extending the Particle Swarm Optimization. Its transfer function is the key element of BPSO. In this paper, a new $\mathrm{V}$-shaped transfer function with a parameter $\mathrm{k}$ has been proposed. The parameter $\mathrm{k}$ was used to control the opening size of the transfer function. At first, the setting of the parameter $\mathrm{k}$ has been obtained by the experiments, and then the new $\mathrm{V}$-shaped transfer with the optimal $\mathrm{k}$ value is compared with the other kinds of the $\mathrm{V}$-shaped transfer functions by the experiment of feature selection. The results have indicated that the new $\mathrm{V}$-shaped transfer function improved the performance of Binary Particle Swarm Optimization.
\end{abstract}

Keywords: Binary Particle Swarm Optimization - V-shaped transfer function - Classification accuracy $\cdot$ Iterative variation of distance between particles

\section{Introduction}

Particle Swarm Optimization (PSO) is an evolutionary computational technique proposed by J. Kennedy and R.C. Eberhart in 1995 [1] and [2]. It is inspired by the social behavior of birds and fish. It uses many particles (candidate solutions) to fly around in the search space to find the best solution. At the same time, they track the best location (best solution) in their own path. In other words, the particles will consider the best solution of their individual and the best solution of the group to get the final best solution. In 1998, Y.Shi and R.C.Eberhart added a weight to control the exploitation and exploration of the PSO algorithm [3]. Because the PSO is simple and computationally inexpensive, it is used in many fields, such as medical diagnosis [4], network scheduling [5], robot path planning [6], wavelength detection [7] and production scheduling [8] and many more.

In order to make the PSO algorithm solve the optimization problem of discrete space, J. Kennedy and R.C. Eberhart proposed a Binary Particle Swarm 
Optimization (BPSO) based on PSO algorithm [9] to solve the optimization problem of discrete space in 1997. In 2008, Yin et al. proposed a new location update formula to improve the BPSO algorithm [10]. In the same year, Wang et al. proposed a new probability mapping formula and applied it to solve the discrete combination of knapsack problems [11]. A key step in BPSO is to require a transfer function to transform the real value to a binary value of 0 or 1. The classical transfer function is an S-shaped transfer function. In 2011, Liu et al. experimentally analyzed the S-shaped transfer function and found the algorithm lack the late local search ability, a V-shaped transfer function, which enhanced the local search ability of the algorithm and effectively improved the performance of the BPSO algorithm [12]. Seyedali et al. also proposed a similar V-shaped transfer function, and compared four S-shaped transfer functions and four $\mathrm{V}$-shaped transfer functions, which proved that the $\mathrm{V}$-shaped transfer function has the advantage of the algorithm [13].

From the above analysis, the $\mathrm{V}$-shaped transfer function is of great significance for the improvement and optimization of the BPSO algorithm. Although the $\mathrm{V}$-shaped transfer function has better performance than the S-shaped transfer function, but what is the best form of the $\mathrm{V}$-shaped transfer function, and how to control the $\mathrm{V}$-shaped transfer function opening size and studied whether the opening size of the $\mathrm{V}$-shaped transfer function has influence on the performance of the algorithm. In this paper proposes a new V-shaped transfer function, which has a parameter $k$ to control the opening size of this $\mathrm{V}$-shaped transfer function, and study the setting of the analysis parameter $k$ algorithm. The parameter $k$ is set to the principle method, and the optimal $k$-value transfer function is compared with other $\mathrm{V}$-shaped transfer functions to verify that the proposed method performs better.

\section{BPSO Algorithm and Its Transfer Function Principle}

Each particle in the BPSO should consider the current position, the current velocity, the distance of the individual best solution pbest, and the distance to the global optimal solution gbest to updata its position. The mathematical model of PSO is shown in Eq.(1) and Eq.(2).

$$
\begin{gathered}
v_{i d}(t+1)=\omega v_{i d}(t)+c_{1} \times \text { rand } \times\left(\text { pbest }-x_{i d}(t)\right)+c_{2} \times \text { rand } \times\left(\text { gbest }-x_{i d}(t)\right) \\
x_{i d}(t+1)=x_{i d}(t)+v_{i d}(t)
\end{gathered}
$$

where $t$ is the current number of iterations, $c_{1}$ and $c_{2}$ are the acceleration coefficients, $v_{i d}(t)$ is the speed of the $i$ th particle in the $d$ th dimension at the $t$ th generation iteration, $w$ is the inertia weight, rand is a random number between 0 and $1, x_{i d}(t)$ is the current position of the $i$ th particle in the $d$ th dimension at the $t$ th generation iteration at iteration $t$, pbest is the best solution obtained by the $i$ th particle in the $d$ th dimension at the $t$ th generation iteration so far, and gbest indicates the best solution obtained by the particle swarm so far. Equation(1) is 
the velocity updating formula of the particle, and Eq.(2) is the position updating formula of the particle.

In order to make the PSO algorithm optimize the problem of discrete binary space and extend the application of PSO algorithm. BPSO's speed updating formula, Eq.(1), is the same as the original PSO algorithm's. But the particle updating formula Eq.(2) is not used for BPSO. In order to make the value of the velocity convert as a probability of binary taking 0 or 1 , the transfer function uses the Sigmoid function of the formula Eq.(3).

$$
s\left(v_{i d}(t)\right)=\frac{1}{1+e^{-v_{i d}(t)}}
$$

The Sigmoid function image is shown a kind of S-shaped function.

After converting the velocity into a probability value, each dimension of position binary vector is updated using its velocity probability formula as Eq.(4).

$$
x_{i d}(t+1)=\left\{\begin{array}{l}
0, \text { if rand } \leq s\left(v_{i d}(t)\right) \\
1, \text { if rand } \geq s\left(v_{i d}(t)\right)
\end{array}\right.
$$

The transfer function is the probability of changing each dimension of the position vector from 0 to 1 , and vice versa. The main function of the transfer function Eq.(3) in the BPSO is to convert the position real value to the probability value between $[0,1]$, and then use the formula Eq.(4) to make the value of the bit two values of $\{0,1\}$. According to Rashedi et al. [14], the design of such a transfer function should consider some principles.

According to the principles proposed by Rashedi et al. it can be found that the sigmoid function is a function with monotonically increaseing and does not meet the rule that the probability of its position changing increases as the absolute value of the speed increases. According to the literature [12], the original BPSO has the too strong global search ability because of using the sigmoid function as its transfer function. Therefore, it is irrational that the BPSO algorithm uses Eq.(3) as a transfer function. Rashedi et al. proposed a kind of V-shaped transfer function with adopting different position update formula as the Eq.(5).

$$
x_{i d}(t+1)= \begin{cases}\left(x_{i d}(t)\right)^{-1}, & \text { if rand }<s\left(v_{i d}(t)\right) \\ x_{i d}(t), & \text { if rand } \geq s\left(v_{i d}(t)\right)\end{cases}
$$

where $\left(x_{i d}(t)\right)^{-1}$ is the complement operator of $x_{i d}(\mathrm{t})$.

Comparing with Eq.(4), Eq.(5) does not force the particles to be changed with the value of 0 or 1 . The transfer function uses a kind of $\mathrm{V}$-shaped function that is called a "V-shaped transfer function." The original V-shaped transfer function proposed by WangLin et al. It can be found that V-shaped transfer function satisfies the principles proposed by Rashedi et al.

$$
s\left(v_{i d}(t)\right)=\left|\tanh \left(v_{i d}(t)\right)\right| .
$$




\section{A New V-Shaped Transfer Function}

According to the above analysis, the V-shaped transfer function has more advantage over the S-shaped transfer function, so the BPSO's transfer function should be a kind of V-shaped function. However, what kind of V-shaped function is appropriate, so this paper does some analytical experiments.

At first, a new V-shaped transfer function is proposed as shown in Eq.(7).

$$
s\left(v_{i d}(t)\right)=1-e^{-k \cdot\left|v_{i d}(t)\right|},
$$

where $k>0$.

The V-shaped transfer function Eq.(7) has a parameter variable $k$, which can control the opening size of the V-shaped transfer function, and can be used to determine the relationship between the opening size of the $\mathrm{V}$-shaped transfer function and the performance of BPSO. Figure 1 demonstrates the opening size of the transfer functions Eq.(7) with different $k$ values. The value of the transfer function with different $k$ is guaranteed to vary between $[0,1]$, and satisfy the principle of the transfer function. As the transfer functions of Eq.(7), parameter $k$ control the opening size of the transfer function as shown in Fig. 1, which can be found that the opening becomes smaller as the value of $k$ increases.

Therefore, the transfer function Eq.(7) with parameter $k$ can be used to analyze performace of BPSO with difference opening size of V-shaped transfer function.

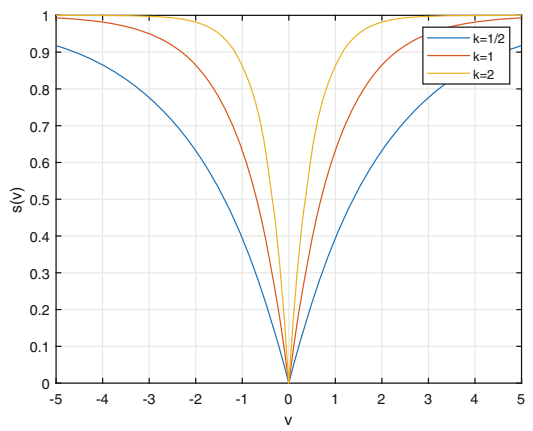

Fig. 1. $\mathrm{k}$ value and the size of the opening

\section{$4 \quad k$ Value Analysis of the Transfer Function}

\subsection{The Average Distance of Particle Swarm}

Definition: The average distance of particle swarm is the average of the distance between each particle in the particle swarm and the global optimal particle, which calculation formula is Eq.(8).

$$
L=\frac{\sum_{i=1}^{n}\left(\sum_{d=1}^{D} g_{d} \bigoplus x_{i d}\right)}{n},
$$


where $L$ represents the average distance of particle swarm which is the value of the average distance of all particles to the global optimal particle, $\bigoplus$ is the logical operator XOR, $n$ is the number of particles, $D$ is the dimension, and $x_{i d}$ is the current position of the $i$ th particle in the $d$ dimension Position, $g_{d}$ is the position of the global optimal particle in the $d$ dimension.

XOR operaor $\bigoplus$ can calculate the number of different binay value beteween two binay vectors. BPSO's particle is a binay code, so $L$ represents the average difference of particles from the global particle. $L$ can be used to measure the algorithm's global exploration and local exploitation capabilities. The larger $L$ is, the stronger the global exploration is, the weaker the local exploitation is. Conversely, the smaller the $L$, the weaker the global exploration and the stronger the local exploitation. According to the basic principle of the swarm intelligence algorithm, the $L$ value should constantly be smaller and smaller. By analyzing the change of the $L$ value over iteration, the relation of the opening size of $\mathrm{V}$ shaped transfer function on the performance of the algorithm can be revealled. Thus Eq.(8) can be used to analyze the principle of setting the value of $k$ for the V-shaped transfer function Eq.(7).

\subsection{The Experimental Analysis on $k$ Value}

For the sake of experimental analysis on $k$ value of Eq.(7), BPSO with the Sshaped transfer function of Eq.(7) is appleid to feature selection.

Feature selection requires classification accuracy of supervised learner as the fitness function of BPSO. K-Nearest Neighbor(KNN) algorithm is a common supervised learner, so KNN is used as the classification algorithm for the feature selection experiment.

The algorithm parameters in the experiment are set as shown in Table 1. The test data for feature selection is six different types of data sets from the UCI machine learning database, which are shown in Table 2.

Table 1. Algorithm parameter settings

\begin{tabular}{l|l|l|l}
\hline $\mathrm{C}_{1}, \mathrm{C}_{2}$ & Total group number & Iterative algebra & KNN parameter $\mathrm{k}$ \\
\hline 2 & 20 & 1000 & 5 \\
\hline
\end{tabular}

The $k$ of transfer function Eq.(7) is set as two group of value, the first group's value are greater than 1 as $\{1.2,3,4,5\}$, the second group's value are less than 1 as $\left\{1, \frac{1}{2}, \frac{1}{3}, \frac{1}{4}, \frac{1}{5}\right\}$. The BPSO algorithm with V-shaped transfer function Eq. (7) using two group of $k$ value is applied for feature selection on datasets in Table 2. During the iteration of BPSO for experimention conducting, $L$ is calculated in each generation of iteration. Figure 2, 3, 4, 5, 6, 7, 8 and 9 show the changes of $L$ over iteration for BPSO to do feature selction for the datasets of Table.2. Each figure of Fig. 2, 3, 4, 5, 6, 7, 8 and 9 include two subfigures which the left subfigure is about $k$ in the first group, the right fiugre is about $k$ in the second group. 
Table 2. Dataset information

\begin{tabular}{l|l|l|l}
\hline Data set & Dimension & Number of samples & Number of categories \\
\hline Snoar & 60 & 208 & 2 \\
\hline SPECTF & 43 & 267 & 2 \\
\hline Lonosphere & 34 & 351 & 2 \\
\hline WDBC & 30 & 569 & 2 \\
\hline SPECT & 22 & 267 & 2 \\
\hline Vehicle & 18 & 846 & 4 \\
\hline Wine & 13 & 178 & 13 \\
\hline Vowel & 10 & 990 & 11 \\
\hline
\end{tabular}
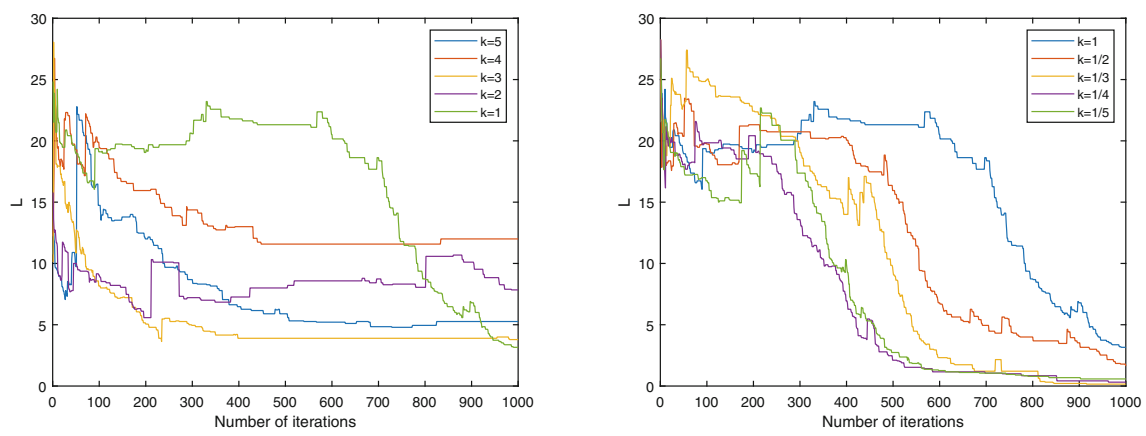

Fig. 2. $L$ change over iteration for selection feature in dataset sonar

Investigating the left subfigures of Fig. 2, 3, 4, 5, 6, 7, 8 and 9, when $k \geq 1$ in the first group, $L$ starts to decrease rapidly, and then tends to be stable quickly over iteration, which indicates that the algorithm are lack of the global exploration and has too strong local exploitation, so BPSO with Eq.(7) is inconsistent with the principle of heuristic random search when $k \geq 1$. Meanwhile, by observing the right subfigures of Fig. 2, 3, 4 and $5, L$ changes from big to small gradually over iteration when $k \leq 1$ which indicates that BPSO algorithm has the strong global exploration ability in the early stage and has strong local exploitation ability in the later stage. Therefore, BPSO with transfer function Eq.(7) satisfies the basical principle of heuristic random search when $k \leq 1$. In one word, the value range of $k$ should be less than or equal to 1 .

\subsection{The Experimental Analysis on BPSO with Different $k$ Values}

In order to decide the appropriate range of $k$, when the experiment are conducted in Sect. 4.2, using transfer function Eq.(7) of with diferent $k$, BPSO do feature selection with KNN learner to classify dataset in Table 2 . The classifing curracy 

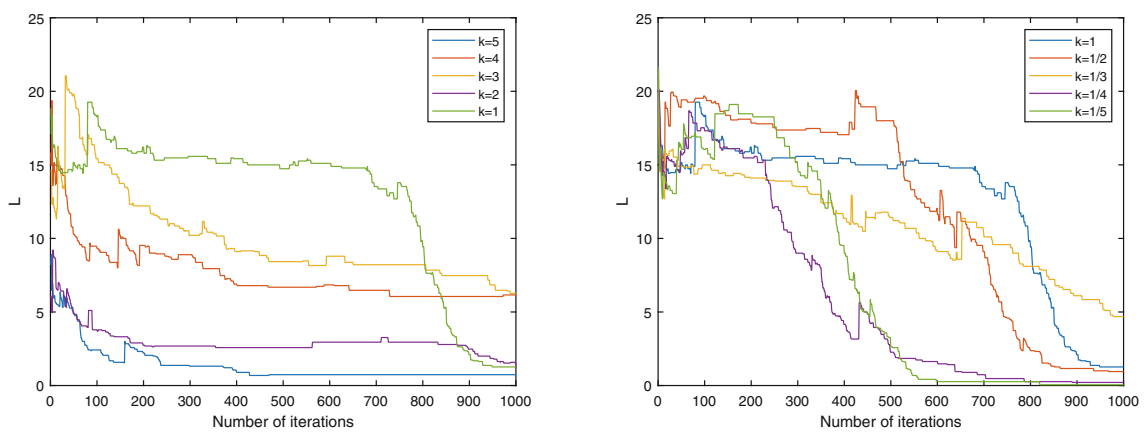

Fig. 3. $L$ change over iteration for selection feature in dataset SPECTF
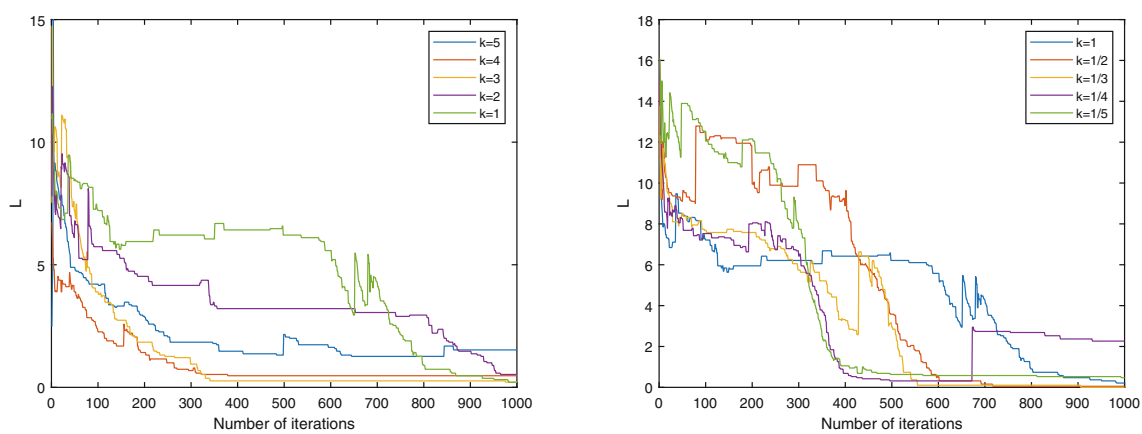

Fig. 4. $L$ change over iteration for selection feature in dataset lonosphere

is used to evalute the performace of BPSO with the V-shaped transfer function of different $k$ so as to investigate which value of $k \geq 1$ is best for transfer function Eq.(7).

For comparison with different $k$, the $k$ value of the transfer function Eq.(7) is set in $\left\{5,4,3,2,1, \frac{1}{2}, \frac{1}{3}, \frac{1}{4}, \frac{1}{5}\right\}$.

BPSO algorithm with each $k$ value transfer function is iteratively run for 1000 generations and runs 5 times. The average of classifing accuracy over 5 times is calculated for each dataset in Table 2. The experimental result are shown in Table 3 .

Dim denotes the dimension of dataset in Table 2. It is found from Table 3 that when the $k>1$, the performance of the BPSO algorithm cannot access to the optimal. The larger the $k$ value is, the worse the performance of the BPSO algorithm is, so $k$ cannot be set as the value which is great to 1 , which is in accordance with the analysis on $L$ in subsection 4.2 . However, when $k \leq 1, k$ becomes smaller and smaller with dimension decreasing, which affects the change of classification accuracy. However, it can be found from Table 3 that the values 

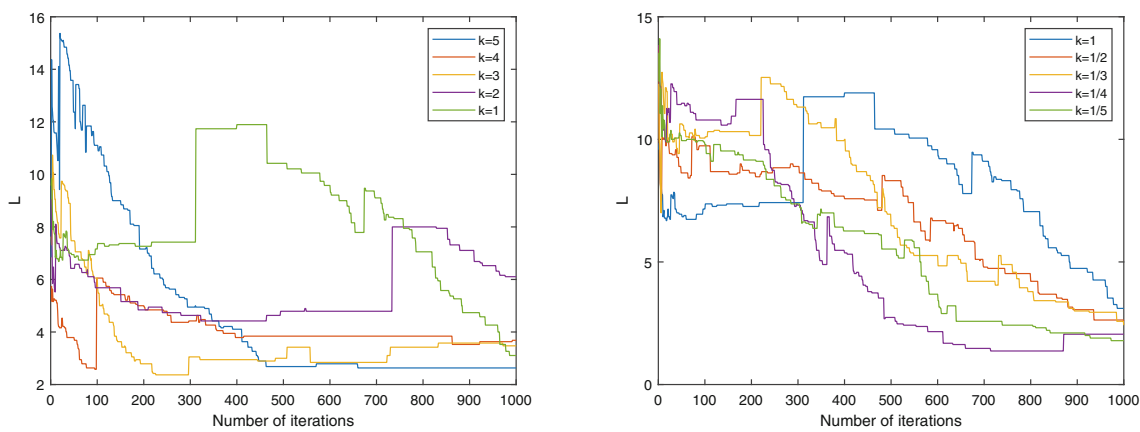

Fig. 5. $L$ change over iteration for selection feature in dataset WDBC
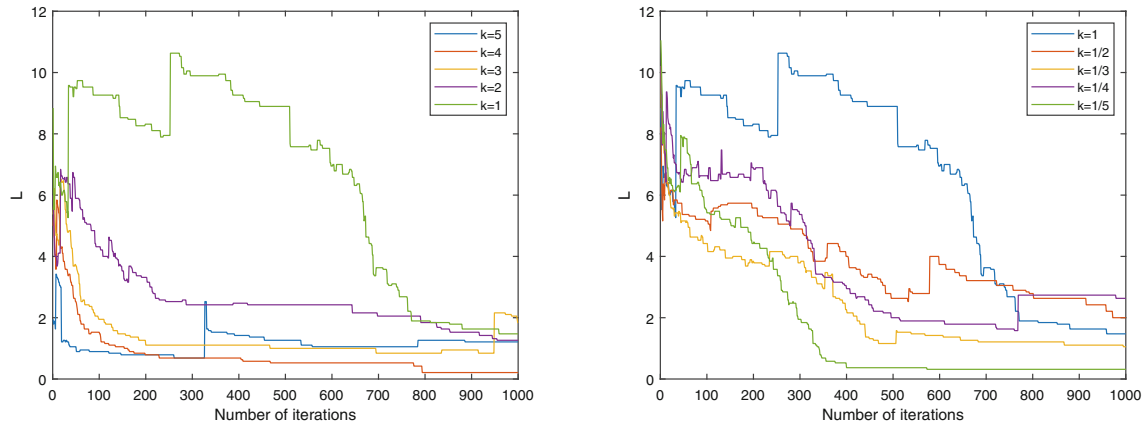

Fig. 6. $L$ change over iteration for selection feature in dataset SPECT
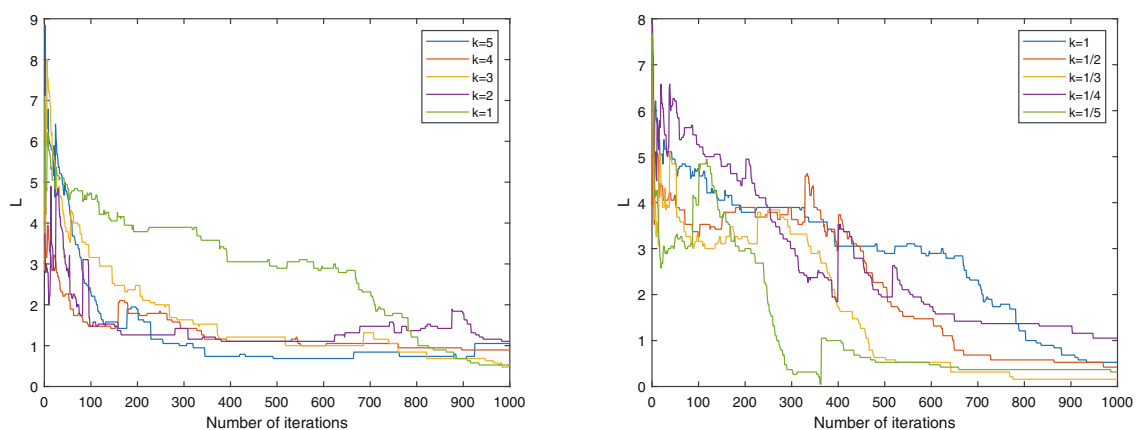

Fig. 7. $L$ change over iteration for selection feature in dataset vehicle

obtained at $\frac{1}{2}$ are relatively high in classification accuracy, so it can be roughly concluded that the opening size of transfer function Eq.(7) will have the highest performance in the transfer function when $k=\frac{1}{2}$. 

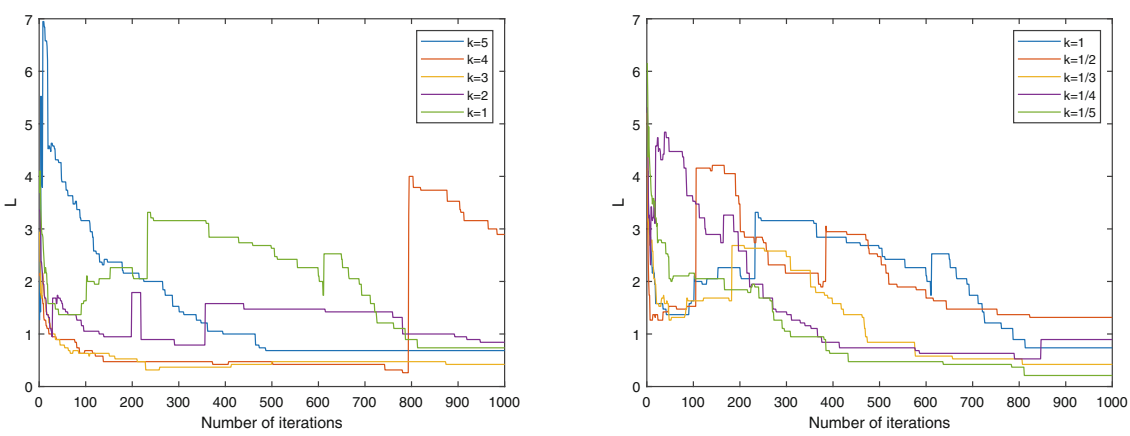

Fig. 8. $L$ change over iteration for selection feature in dataset wine
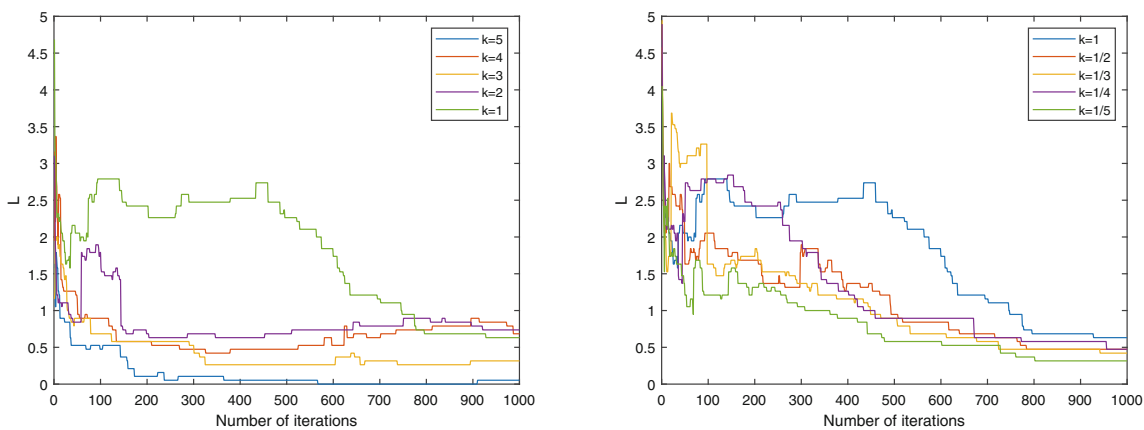

Fig. 9. $L$ change over iteration for selection feature in dataset vowel

Table 3. Classification accuracy of feature selection for different $\mathrm{k}$ value

\begin{tabular}{l|l|l|l|l|l|l|l|l|l}
\hline Dim & 5 & 4 & 3 & 2 & 1 & $\frac{1}{2}$ & $\frac{1}{3}$ & $\frac{1}{4}$ & $\frac{1}{5}$ \\
\hline 60 & 0.9178 & 0.9217 & 0.9231 & 0.9298 & 0.9399 & $\mathbf{0 . 9 5 4 8}$ & 0.9442 & 0.9399 & 0.9447 \\
\hline 43 & 0.8545 & 0.8611 & 0.8503 & 0.8623 & 0.8689 & $\mathbf{0 . 8 8 2 3}$ & 0.8775 & 0.8794 & 0.8805 \\
\hline 34 & 0.9208 & 0.9219 & 0.9253 & 0.9288 & 0.9362 & 0.9402 & 0.9425 & $\mathbf{0 . 9 4 1 9}$ & 0.9385 \\
\hline 30 & 0.9821 & 0.9845 & 0.9831 & 0.9835 & 0.9849 & 0.9852 & 0.9859 & $\mathbf{0 . 9 8 6 3}$ & 0.9849 \\
\hline 22 & 0.8734 & 0.8786 & 0.8771 & 0.8786 & 0.8809 & $\mathbf{0 . 8 8 1 6}$ & 0.8816 & 0.8816 & 0.8831 \\
\hline 18 & 0.7896 & 0.7915 & 0.7943 & 0.7950 & 0.7948 & 0.7981 & 0.7999 & 0.7995 & $\mathbf{0 . 8 0 0 0}$ \\
\hline 13 & 0.9955 & 0.9955 & 0.9966 & 0.9978 & 0.9955 & $\mathbf{0 . 9 9 8 9}$ & 0.9966 & 0.9978 & 0.9978 \\
\hline 10 & 0.9519 & 0.9485 & 0.9496 & 0.9508 & 0.9523 & 0.9508 & 0.9515 & $\mathbf{0 . 9 5 3 8}$ & 0.9519 \\
\hline
\end{tabular}

\section{Conclusion}

In this paper, the opening size of the $\mathrm{V}$-shaped transfer function of the discrete binary particle swarm optimization algorithm is studied. The experimen- 
tal results of the classification accuracy and the iterative variation between the particles by the $\mathrm{V}$-shaped transfer function with different opening sizes indicate that the optimal point of the performance can be obtained when the parameter of the control opening size $k$ is $\frac{1}{2}$.

\section{References}

1. Eberhart, R., Kennedy, J.: A new optimizer using particle swarm theory. In: MHS 1995 Sixth International Symposium on Micro Machine Human Science (2002)

2. Kennedy, J., Eberhart, R.: Particle swarm optimization. In: ICNN 1995International Conference on Neural Networks (2002)

3. Shi, Y.H., Eberhart, R.C.: A modified particle swarm optimizer. In: 1998 IEEE International Conference on Evolutionary Computation Proceedings. IEEE World Congress on Computational Intelligence. IEEE (1998)

4. Chandra, S., Bhat, R., Singh, H.A.: PSO based method for detection of brain tumors from MRI. In: 2009 World Congress on Nature Biologically Inspired Computing (NABIC). IEEE (2010)

5. Martino, V.D., Mililotti, M.: Scheduling in a grid computing environment using genetic algorithms. In: IPDPS 2002 Proceedings of the International Parallel and Distributed Processing Symposium (2002)

6. Masehian, E., Sedighizadeh, D.: A multi-objective PSO-based algorithm for robot path planning. In: 2010 IEEE International Conference on Industrial Technology. IEEE (2010)

7. Liang, J.J., Suganthan, P.N., Chan, C.C., et al.: Wavelength detection in FBG sensor network using tree search DMS-PSO. IEEE Photonics Technol. Lett. 18(12), 1305-1307 (2006)

8. Liu, J., Fan, X., Qu, Z.: An improved particle swarm optimization with mutation based on similarity. In: International Conference on Natural Computation (2007)

9. Kennedy, J., Eberhart, R.C.: A discrete binary version of the particle swarm algorithm. In: IEEE International Conference on Systems (2002)

10. Yin, P.Y.: A discrete particle swarm algorithm for optimal polygonal approximation of digital curves. J. Vis. Commun. Image Represent. 15(2), 241-260 (2004)

11. Zhen, L., Wang, L., Huang, Z.: Probability-based binary particle swarm optimization algorithm and its application to WFGD control. In: International Conference on Computer Science Software Engineering (2008)

12. Jianhua, L., Ronghua, Y., Shuihua, S.: Analysis of discrete binary particle swarm optimization algorithm. J. Nanjing Univ. Nat. Sci. 5, 504-514 (2011)

13. Mirjalili, S., Lewis, A.: S-shaped versus V-shaped transfer functions for binary particle swarm optimization. Swarm Evol. Comput. 9, 1-14 (2013)

14. Rashedi, E., Nezamabadi-Pour, H., Saryazdi, S.: BGSA: binary gravitational search algorithm. Nat. Comput. 9(3), 727-745 (2010)

15. Cover, T.M., Hart, P.: Nearest neighbor pattern classification. In: Proceeding of the IEEE Transactions Information Theory, pp. 21-27 (1967)

16. Kang, L., Minrui, F., Li, J., Irwin, G.W. (eds.): ICSEE/LSMS -2010. LNCS, vol. 6329. Springer, Heidelberg (2010). https://doi.org/10.1007/978-3-642-15597-0 